\title{
Bioactive proanthocyanidins inhibit growth and induce apoptosis in human melanoma cells by decreasing the accumulation of $\boldsymbol{\beta}$-catenin
}

\author{
MUDIT VAID $^{1}$, TRIPTI SINGH ${ }^{1,4}$, RAM PRASAD ${ }^{1,4}$ and SANTOSH K. KATIYAR ${ }^{1-4}$ \\ ${ }^{1}$ Department of Dermatology, ${ }^{2}$ Comprehensive Cancer Center, ${ }^{3}$ Nutrition Obesity Research Center, University of \\ Alabama at Birmingham, AL 35294; ${ }^{4}$ Birmingam Veterans Affairs Medical Center, Birmingham, AL 35233, USA
}

Received September 25, 2015; Accepted October 23, 2015

DOI: 10.3892/ijo.2015.3286

\begin{abstract}
Melanoma is a highly aggressive form of skin cancer with poor survival rate. Aberrant activation of $\mathrm{Wnt} / \beta$-catenin has been observed in nearly one-third of human melanoma cases thereby indicating that targeting Wnt/ $/$-catenin signaling could be a promising strategy against melanoma development. In the present study, we determined chemotherapeutic effect of grape seed proanthocyanidins (GSPs) on the growth of melanoma cells and validated their protective effects in vivo using a xenograft mouse model, and assessed if $\beta$-catenin is the target of GSP chemotherapeutic effect. Our in vitro data show that treatment of A375 and Hs294t human melanoma cells with GSPs inhibit the growth of melanoma cells, which was associated with the reduction in the levels of $\beta$-catenin. Administration of dietary GSPs $(0.2$ and $0.5 \%$, w/w $)$ in supplementation with AIN76A control diet significantly inhibited the growth of melanoma tumor xenografts in nude mice. Furthermore, dietary GSPs inhibited the xenograft growth of Mel928 ( $\beta$-catenin-activated), while did not inhibit the xenograft growth of Mel1011 ( $\beta$-catenin-inactivated) cells. These observations were further verified by siRNA knockdown of $\beta$-catenin and forced overexpression of $\beta$-catenin in melanoma cells using a cell culture model.
\end{abstract}

\section{Introduction}

Melanoma, a cancer originating from melanocytes, is one of the most aggressive forms of skin cancers with a high frequency of metastasis. Estimates suggest that 76,690 new cases of melanoma were diagnosed in year 2013 in USA alone, leading to $\sim 9480$ deaths. Solar ultraviolet (UV) radiation

Correspondence to: Dr Santosh K. Katiyar, Department of Dermatology, University of Alabama at Birmingham, 1670 University Boulevard, Volker Hall 557, Birmingham, AL 35294, USA

E-mail: skatiyar@uab.edu

Key words: grape seed proanthocyanidins, melanoma, $\beta$-catenin, apoptosis, tumor xenograft exposure is a recognized risk factor for the development of cutaneous malignancies including the risk of melanoma. The incidence of melanoma is increasing in US and is increasing rapidly in children $(1,2)$ because of the increase in recreational UV light exposure. Although melanoma is less common than non-melanoma, however, majority of skin cancer-related deaths are due to melanoma. As, melanoma is a highly malignant cancer with a potent capacity to metastasize to distant organs, an approach that inhibits its growth and progression may facilitate the development of an effective strategy for its prevention or treatment.

Natural plant products offer promising tools for the prevention of cancer growth and metastasis. Grape seed proanthocyanidins (GSPs) are bioactive phytochemicals that have shown strong anti-carcinogenic activity in various animal tumor models and appear to exhibit minimal toxicity in laboratory animals $(3,4)$. GSPs are readily extracted from grape-seeds, and are a mixture of dimers, trimers, tetramers, and oligomers of monomeric catechins and/or (-)-epicatechins $(3,4)$. GSPs have been shown to have anti-inflammatory, anti-oxidant and anti-metastatic properties in both in vitro and in vivo models (3-7). They inhibit UV radiation- and chemical carcinogen-induced skin carcinogenesis in mouse models $(3,8)$. Dietary administration of GSPs resulted in a dosedependent inhibition of the growth of tumor xenografts of cancer cells of lungs (9), pancreas (10) and head and neck (11). Recently, we showed that GSPs inhibit the invasive potential of melanoma cells (6). However, the anticarcinogenic potential of GSPs against melanoma growth and progression is largely unexplored.

$\beta$-catenin, a key component of Wnt signaling pathway, is a complicated dual function protein. It participates in formation of adherens junctions via formation of a stable complex with the cell adhesion proteins of the cadherin family, while in free non-phosphorylated state, $\beta$-catenin interacts with the T-cell factor transcription factors to control expression of target genes that are involved in cell proliferation, differentiation and metastasis. Though various studies have implicated nuclear accumulation of $\beta$-catenin occurring as a result of constitutively active Wnt/ $\beta$-catenin signaling in growth and progression of cancers of various organs (12-14), the view that $\beta$-catenin is 'uniformly oncogenic' is far from acceptable in the 
scientific community. Studies have shown that forced expression of a melanocyte-specific, non-degradable, constitutively active $\beta$-catenin mutant in either transgenic or Cre/lox systems is not sufficient enough to induce melanoma in mice (15). Most importantly studies in human melanoma patients suggest a positive correlation between increased levels of nuclear $\beta$-catenin and an improved rather than poorer prognosis of melanoma indicate that $\mathrm{Wnt} / \beta$-catenin signaling may not be oncogenic, but rather is required to prevent early melanoma transformation (14,16-19). Overall, in view of limited information concerning $\beta$-catenin, the oncogenic/tumor suppressive role of $\beta$-catenin in case of melanoma may best be regarded as contextual i.e. dependent on the model system employed for the study.

In the present study, we determined growth inhibitory effect of GSPs on melanoma using two different human melanoma cell lines, namely A375 (BRAF-mutated) and Hs294t (wild-type for BRAF gene, non-BRAF-mutated). For this purpose both in vitro and in vivo tumor xenograft models were used. Results of the present study indicate a pro-oncogenic role of $\beta$-catenin in melanoma and also suggest that GSPs inhibit melanoma growth by targeting $\beta$-catenin in our model system.

\section{Materials and methods}

Chemicals and antibodies. The purified fraction of proanthocyanidins from grape seeds were obtained from the Kikkoman Corp. (Noda, Japan). The $\beta$-catenin ${ }^{\mathrm{S} 33 \mathrm{Y}}$ pcDNA plasmid bearing FLAG tag used for the overexpression of non-degradable, constitutively active mutant form of $\beta$-catenin was obtained from Addgene (Cambridge, MA, USA), while $\beta$-catenin siRNA kit for knocking down the expression level of $\beta$-catenin along with the siRNA transfection reagents, and antibodies specific to PCNA, cyclin D1, cyclin D2, Cdks $(2,4,6), \mathrm{Cip} 1 / \mathrm{p} 21, \mathrm{Kip} 1 / \mathrm{p} 27, \beta$-catenin, $\beta$-actin, histone H3, horseradish peroxidase conjugated rabbit anti-goat and goat anti-rabbit secondary antibodies were purchased from Santa Cruz Biotechnology (Santa Cruz, CA, USA). The antibodies specific for Bax, Bcl-2, Bcl-xl, cleaved caspase-3, caspase-9, PARP, casein kinase $1 \alpha(\mathrm{CK} 1 \alpha)$, glycogen synthase kinase-3 $\beta$ (GSK-3 $\beta$ ), and phospho forms of $\beta$-catenin were obtained from Cell Signaling Technology (Beverly, MA, USA). Annexin V-conjugated Alexa Fluor 488 apoptosis detection kit was purchased from Molecular Probes, Inc. (Eugene, OR, USA).

Cell lines and cell culture conditions. The human melanoma cells lines, Mel928, Mel1011 and Mel1241, were a kind gift from Dr Paul Robbins (Center of Cancer Research, National Cancer Institute, Bethesda, MD, USA), while A375 and Hs294t human melanoma cell lines were purchased from the American Type Culture Collection (ATCC; Manassas, VA, USA). No further authentication of cells was done by the authors. Cells were grown in cell culture media as previously detailed (6). For treatment of cells, GSPs were dissolved in a small amount $(100 \mu \mathrm{l})$ of dimethylsulfoxide (DMSO), which was then added to the complete cell culture medium. The maximum concentration of DMSO in cell culture media was not $>0.1 \%(\mathrm{v} / \mathrm{v})$. Cells treated with the same concentration of DMSO only served as a vehicle control.
Cell viability assay or MTT assay. The effect of GSPs on the viability of human melanoma cells was determined using MTT assay as previously described (20). The effect of GSPs on cell viability was calculated and presented in terms of percent of control, which was arbitrarily assigned a value of $100 \%$ viability. All treatment concentrations were replicated at least in 6-wells.

Apoptotic cell death analysis by flow cytometry. GSPs induced apoptotic cell death in melanoma cells was quantitatively determined by flow cytometry using the Annexin V-conjugated Alexa Fluor 488 (Alexa 488) apoptosis detection kit following the manufacturer's protocol, as was previously used and described (20). Briefly, after treatment of cells with GSPs $(0,20,40$ and $60 \mu \mathrm{g} / \mathrm{ml})$ for $48 \mathrm{~h}$, cells were harvested, washed with PBS and stained with Alexa 488 and propidium iodide. The cells were then analyzed by fluorescence activated cell sorting using the FACSCalibur instrument (BD Biosciences, San Jose, CA, USA) and CellQuest 3.3 software, as previously described (20).

siRNA knockdown of $\beta$-catenin in melanoma cells. The $\beta$-catenin expression was knocked down in Mel1241 melanoma cells using $\beta$-catenin siRNA kit obtained from Santa Cruz Biotechnology following the manufacturer's instruction. Briefly, Mel1241 cells (3x105/well) were seeded in a 6-well plate and allowed to grow to $70 \%$ confluency. The $\beta$-catenin siRNA mixed with transfection reagents was overlaid on the cells for $6 \mathrm{~h}$ at $37^{\circ} \mathrm{C}$ and transferred into $2 \mathrm{X}$ growth medium for $\sim 18-20 \mathrm{~h}$. At $24 \mathrm{~h}$ post-transfection, fresh medium was added to the cells and the cells were incubated for an additional $48 \mathrm{~h}$. The knockdown of $\beta$-catenin expression in cells after transfection was confirmed by western blot analysis using $\beta$-catenin-specific antibody.

Forced-overexpression of mutant $\beta$-catenin in melanoma cells. For the forced overexpression of mutant $\beta$-catenin, expression plasmid ( $\beta$-catenin ${ }^{\mathrm{S} 33 \mathrm{Y}}$ pcDNA) was transfected in Mel1011 cells (inactivated $\beta$-catenin cell line) using Lipofectamine 2000 (Invitrogen, Carlsbad, CA, USA) following the manufacturer's protocol and stable clones were obtained through $1000 \mu \mathrm{g} / \mathrm{ml}$ G418 (Invitrogen) selection. Mutant forced- $\beta$-catenin overexpression was checked by western blotting using monoclonal anti-FLAG antibody (Sigma, St. Louis, MO, USA).

Western blot analysis. Following treatment of melanoma cells with or without GSPs the cells were harvested, cell lysates were prepared using ice-cold lysis buffer supplemented with protease inhibitors, as previously detailed (20). In case of tumor xenografts, tumor lysates were also prepared following the same procedure after homogenizing the tumor samples in lysis buffer. Western blot analysis was conducted as previously detailed $(11,20)$. The membrane was stripped and re-probed with anti- $\beta$-actin antibody to verify equal protein loading on the gel.

Athymic nude mice and tumor xenograft model. Female athymic nude mice of 4-5 weeks of age were purchased from the National Cancer Institute (NCI; Bethesda, MD, USA) and housed in the Animal Resource Facility at the University of 
Alabama at Birmingham in accordance with the Institutional Animal Care and Use Committee (IACUC) guidelines. The mice were given control AIN76A diet with or without supplementation with GSPs $(0.2$ and $0.5 \%, \mathrm{w} / \mathrm{w})$ and drinking water $a d$ libitum throughout the experiment. The animal protocol used in the present study was approved by the IACUC. To determine the in vivo chemotherapeutic efficacy of GSPs against tumor xenograft growth, exponentially growing A375 cells $\left(3 \times 10^{6}\right.$ in $100 \mu \mathrm{l}$ PBS) or other cell lines were injected subcutaneously in the right flank of each mouse. One day after tumor cell inoculation, mice were divided randomly into different treatment groups with five mice per group. One group of mice received the AIN76A control diet, while other groups of mice received a 0.2 or $0.5 \%$ (w/w) GSPs-supplemented AIN76A control diet in pellet throughout the experiment protocol. The tumor growth/size and body weight of each mouse was recorded on weekly basis. Tumor size was measured using Vernier calipers and volumes were calculated using the hemiellipsoid model formula: tumor volume $=1 / 2(4 \pi / 3)(1 / 2)(w / 2) h$, where 1 is the length, $w$ is the width and $h$ is the height. At the termination of the experiment, mice were sacrificed, tumor from each mouse was excised and the wet weight of each tumor was recorded on digital balance. A part of the tumor was used to prepare tumor lysates for western blot analysis and the other part of the tumor tissues was paraffin-embedded and used for immunohistochemical analysis.

Immunohistochemical detection and analysis of $\mathrm{PCNA}^{+}$and activated caspase- $3^{+}$cells. Paraffin-embedded tumor sections (5 $\mu \mathrm{m}$ thick) were deparaffinized, rehydrated and then antigen retrieval was carried out, as previously described (21). The non-specific binding sites were blocked with $3 \%$ bovine serum albumin in PBS buffer before incubation with anti-PCNA or anti-cleaved caspase-3 specific antibodies. After washing, the sections were incubated with biotinylated secondary antibody followed by horseradish peroxidase-conjugated streptavidin. The sections were further incubated with 2,4-diaminobenzidine substrate and counterstained with hematoxylin. The PCNA-positive and activated caspase-3-positive positive cells in sections were counted in at least 4-5 different fields and photographed using an Olympus microscope (Model BX40F4; Olympus Tokyo, Japan) fitted with a Q-Color 5 Olympus camera.

Statistical analysis. The statistical significance of the difference between the values of control and treatment groups was determined by either Student's t-test or simple one-way ANOVA using GraphPad Prism version 4.00 for Windows, (GraphPad software, San Diego, CA, USA). In each case, $\mathrm{P}<0.05$ was considered statistically significant.

\section{Results}

GSPs exert cytotoxic effects on melanoma cells and inhibit their viability. The cytotoxic effects of GSPs on melanoma cells, A375 and Hs294t, were determined using MTT assay. As shown in Fig. 1A, compared with the non-GSP-treated control cells, treatment of A375 cells with GSPs for 24 and $48 \mathrm{~h}$ resulted in a significant dose-dependent decrease in cell viability. Treatment for $24 \mathrm{~h}$ resulted in a $4-20 \%$ decrease in cell viability, while treatment for $48 \mathrm{~h}$ resulted in $11-57 \%$ $(\mathrm{P}<0.01-0.001)$ decrease in cell viability. Under identical conditions, GSPs exerted similar inhibitory effects on the viability of Hs294t cells, as shown in Fig. 1B. However, the growth of normal human epidermal melanocytes was not significantly affected by GSPs-treatment under identical experimental conditions

GSPs induce apoptosis in human melanoma cells. To examine whether inhibition of cell viability in A375 and Hs294t melanoma cells by GSPs is due to the induction of apoptosis in these cells, the A375 and Hs294t cells were treated with varying concentrations of GSPs $(0,20,40$ and $60 \mu \mathrm{g} / \mathrm{ml})$ for $48 \mathrm{~h}$ and apoptotic cells were analyzed using the Alexa 488/PI apoptotic cell detection kit. Apoptotic cell death was determined in terms of early-stage and late-stage apoptotic cells, which are shown respectively in the lower right (LR) and upper right (UR) quadrants of the FACS histograms (Fig. 1C). Treatment of the A375 and Hs294t cells with GSPs for $48 \mathrm{~h}$ resulted in significant induction of apoptosis in both cell lines. The percentage of total apoptotic cells (in UR+LR quadrants) in A375 cells after treatment with GSPs was as follows: $4.5 \%$ (vehicle-treated control), 11.8\% (20 $\mu \mathrm{g} / \mathrm{ml}, \mathrm{P}<0.05), 16.5 \%$ (40 $\mu \mathrm{g} / \mathrm{ml}, \mathrm{P}<0.01), 30.3 \%(60 \mu \mathrm{g} / \mathrm{ml}, \mathrm{P}<0.001)$ as summarized in Fig. 1D. Similarly, GSP-induced apoptosis was also observed when Hs294t cells were treated with GSPs for 48 h, as shown in Fig. 1C and D.

GSPs affect the protein expression of Bcl-2 family and activate caspase-3 and poly (ADP-ribose) polymerase (PARP) in human melanoma cells. The proteins of the Bcl-2 family play critical roles in regulation of apoptosis by acting as promoters or inhibitors of cell death process $(22,23)$. Therefore, the effect of GSPs on the proteins of Bcl-2 family in both A375 and Hs294t cells was determined. For this purpose, A375 and Hs $294 \mathrm{t}$ cells were treated with GSPs $(0,20,40$ and $60 \mu \mathrm{g} / \mathrm{ml})$ for $48 \mathrm{~h}$, and cell lysates were prepared and subjected to western blot analysis. Western blot analysis revealed that treatment of cells with GSPs resulted in a dose-dependent reduction in the levels of the $\mathrm{Bcl}-2$ and $\mathrm{Bcl}-\mathrm{xl}$ proteins with a concomitant increase in the levels of Bax compared with the cells that were not treated with GSPs (Fig. 1E). These data indicate that GSPs treatment can alter the protein levels of key members of the Bcl-2 family in a manner that contribute to the susceptibility of melanoma cells to GSP-induced apoptosis (Fig. 1E). Western blot analysis also revealed that treatment of A375 and Hs294t cells with GSPs enhanced the activation or cleavage of caspase-3 and PARP when compared with the cells which were not treated with GSPs (Fig. 1E). These events contribute to the induction of cancer cell apoptosis.

GSPs reduce cellular accumulation of $\beta$-catenin in melanoma cells. Studies have indicated that aberrant activation of Wnt signaling leading to $\beta$-catenin overexpression or accumulation is involved in cancers of various organs including melanoma. Therefore, we determined the effect of GSPs on the levels of $\beta$-catenin protein in both A375 and Hs294t cells using western blot analysis. For this purpose melanoma cells were treated with GSPs for $48 \mathrm{~h}$ and cytosolic and nuclear fractions were prepared. Western blot analysis revealed that treatment of 
A
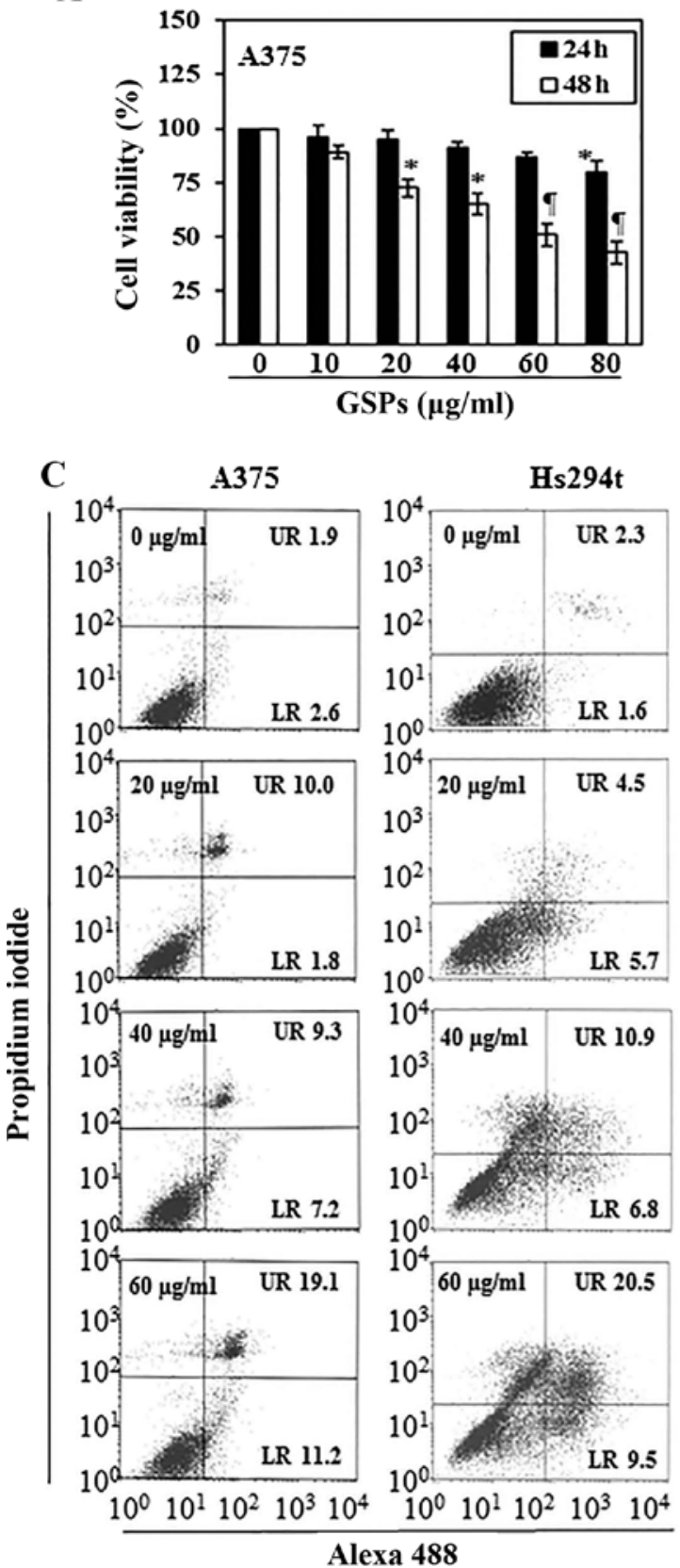

B

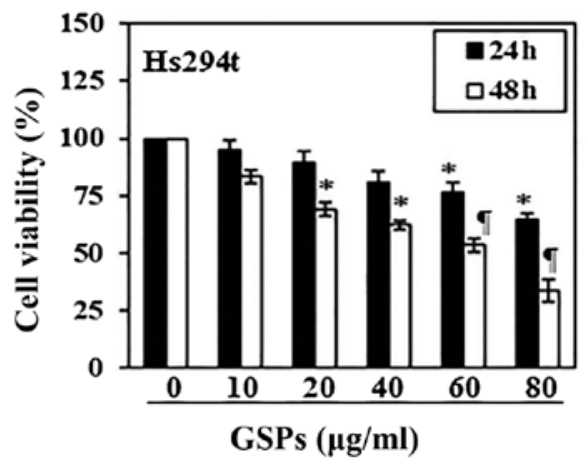

D
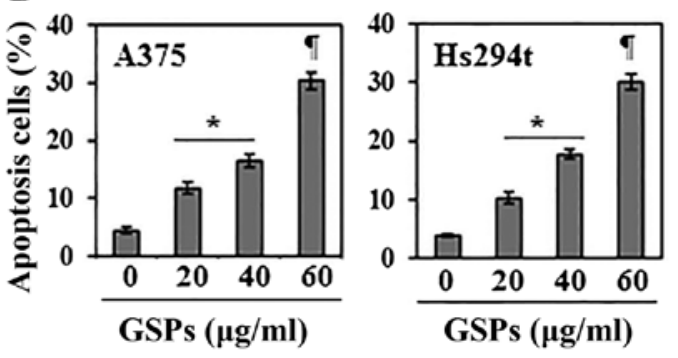

$\mathbf{E}$

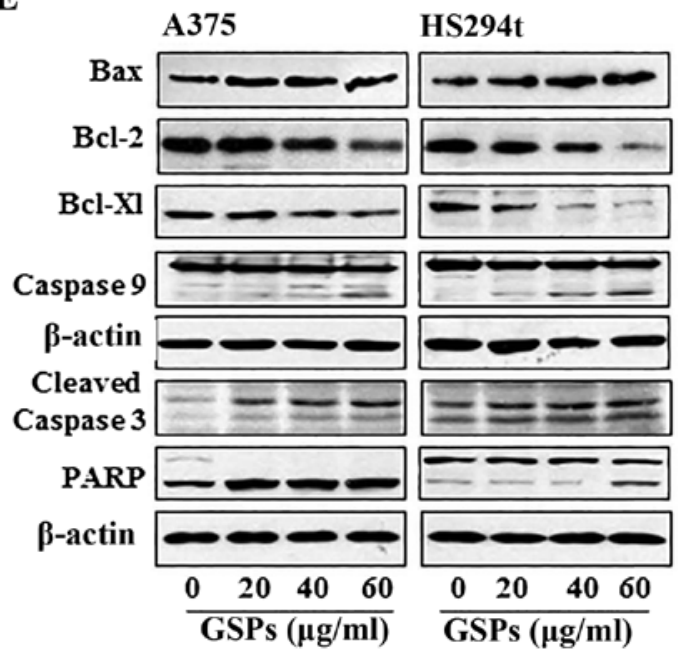

Figure 1. Treatment of human melanoma cells with GSPs inhibits cell viability and induce apoptotic cell death. (A and B) A375 and Hs294t cells were treated with varying concentrations of GSPs for 24 and $48 \mathrm{~h}$ and cell viability was determined using the MTT assay, as described in Materials and methods. Cell viability is expressed in terms of percent of control (non-GSPs-treatment), as the mean $\pm \mathrm{SD}, \mathrm{n}=6-8$. " $\mathrm{P}<0.01$; ${ }^{\mathrm{I}} \mathrm{P}<0.001$ vs. non-GSP-treated control group. (C) Melanoma cells, A375 and Hs294t, were treated with varying concentrations of GSPs $(0,20,40$ and $60 \mu \mathrm{g} / \mathrm{ml})$ for $48 \mathrm{~h}$, then harvested for the analysis of apoptosis using the Annexin V-Alexa Fluor 488 (Alexa 488) apoptosis vybrant assay kit. (D) The percentage of apoptotic cells in each treatment group summarized after $48 \mathrm{~h}$ of GSPs treatment. Data are presented as mean \pm SD of three experiments. Significant difference vs. control group (non-GSP-treated). ${ }^{*} \mathrm{P}<0.01$ and ${ }^{\mathrm{I}} \mathrm{P}<0.001$. (E) Western blot analysis of pro-apoptotic and anti-apoptotic proteins in A375 and Hs294t cells.

A375 and Hs294t cells with GSPs resulted in reduced expression levels of $\beta$-catenin in both nucleus as well as in cytoplasm of the cells (Fig. 2A). Since, cellular accumulation of $\beta$-catenin is inversely correlated with phosphorylation at certain key residues of $\beta$-catenin $\left(\mathrm{Ser}^{45}, \mathrm{Ser}^{33}, \mathrm{Ser}^{37}\right.$ and $\mathrm{Thr}^{41}$ ), we checked the effect of GSPs on the levels of $\beta$-catenin phosphorylation at these sites. Western blot analysis of the cytoplasmic fraction of melanoma cells revealed that treatment of A375 and Hs294t cells with GSPs increased the phosphorylation of $\beta$-catenin at $\mathrm{Ser}^{45}$, and $\mathrm{Ser}^{33} / \mathrm{Ser}^{37} / \mathrm{Thr}^{41}$ in both melanoma cell lines
(Fig. 2A). Furthermore, GSP treatment of melanoma cells resulted in a dose-dependent increase of CK1 $\alpha$ and GSK-3 $\beta$. Both CK $1 \alpha$ and GSK-3 $\beta$ are known to target $\beta$-catenin for proteasomal degradation via combined phosphorylation at key residues of $\beta$-catenin (24). These results suggest that GSPs enhance melanoma cell apoptosis by targeting $\beta$-catenin in both A375 and Hs294t cells.

To further evaluate if $\beta$-catenin is a molecular target of GSPs, we used melanoma cell lines that differ in the activation status of $\beta$-catenin and compared the cytotoxic effects of 
A

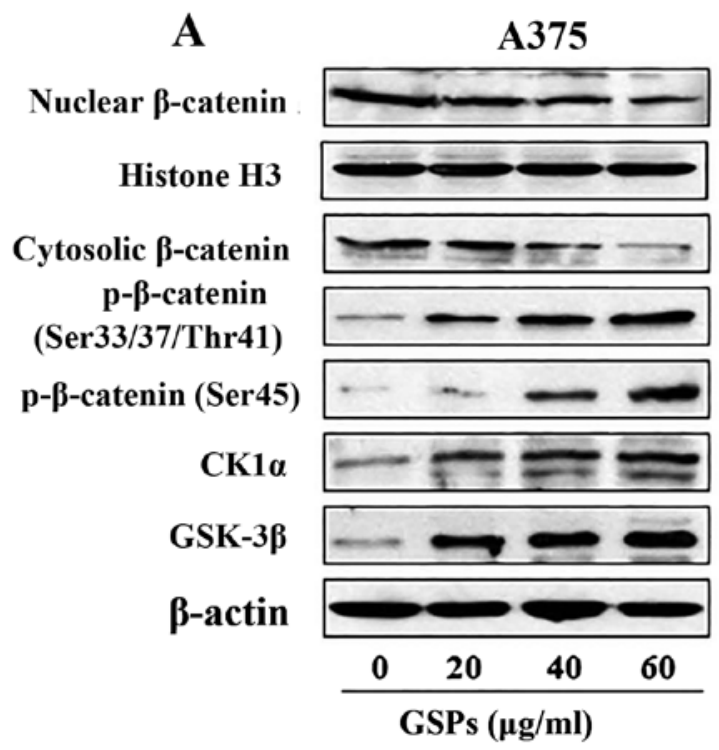

Hs294t

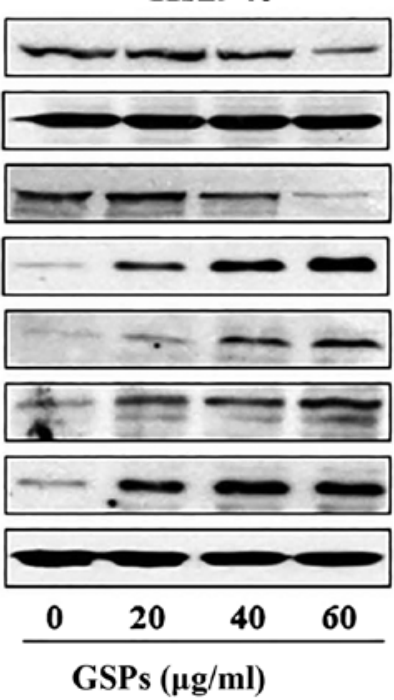

B
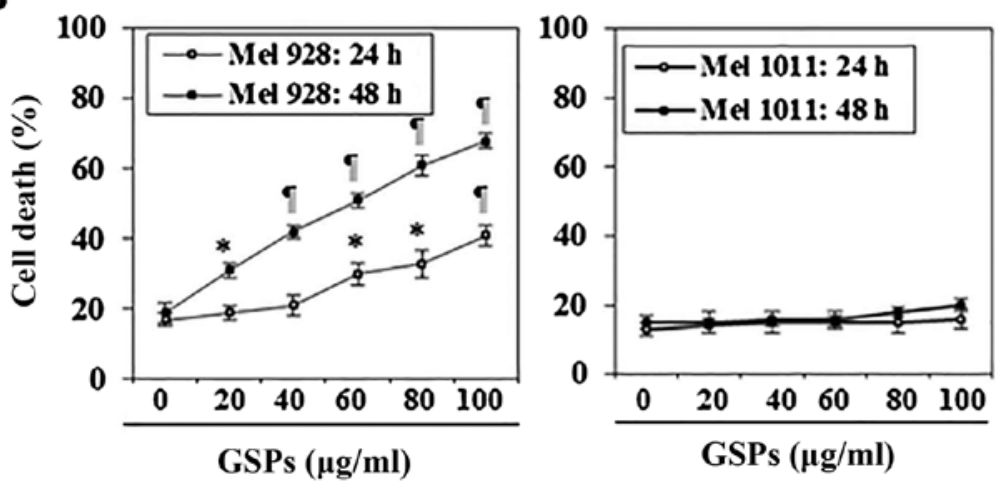

Figure 2. Effect of GSPs on $\beta$-catenin and its signaling molecules in human melanoma cells in vitro. (A) A375 and Hs294t cells were treated with varying concentrations of GSPs for $48 \mathrm{~h}$. Cell lysates were subjected to the western blot analysis of different proteins of $\beta$-catenin signaling pathway. (B) Effect of GSPs on cell death of Me1928 cells (activated $\beta$-catenin) vs. Mel1011 cells (wt $\beta$-catenin) as analyzed by standard trypan blue staining. Cells were treated with GSPs for 24 and $48 \mathrm{~h}$, then harvested. The number of dead melanoma cells were counted under a microscope. Resultant dead cells in each treatment group are expressed as a percentage of total number of cells/treatment group. Data shown are mean $\pm \mathrm{SD}, \mathrm{n}=3$. ${ }^{*} \mathrm{P}<0.01$; ${ }^{\text {I }} \mathrm{P}<0.001$ vs. non-GSP-treated control group.

GSPs. For this purpose Mel928 and Mel1011 cell lines were treated with GSPs $(0,20,40,60,80$ and $100 \mu \mathrm{g} / \mathrm{ml})$ for 24 and $48 \mathrm{~h}$. Cells were harvested and cytotoxicity of GSPs was evaluated using trypan blue staining assay. Mel928 cells exhibit constitutively activated $\beta$-catenin signaling, while Mel1011 cells exhibit inactivated $\beta$-catenin. As shown in Fig. $2 \mathrm{~B}$, treatment of Mel928 cells with GSPs significantly enhanced cell death $(\mathrm{P}<0.01-0.001)$ in a dose- and time-dependent manner. Resultant data on cell death is summarized in terms of percentage of dead cells \pm SD for different treatment groups. However, GSPs failed to exert significant cytotoxic effects in Mel1011 cells (Fig. 2B, right panel).

Dietary GSPs inhibit the growth of tumor xenograft of melanoma cells in nude mice. Next, we sought to determine whether dietary administration of GSPs inhibit in vivo tumor xenograft growth in a nude mouse model. As the growth inhibitory effect of GSPs on both A375 and Hs294t cell lines were identical, we selected the A375 cell line for further in vivo studies. The mice were given the AIN76A control diet alone or the same diet supplemented with GSPs $(0.2$ and $0.5 \%$, w/w). The dietary doses of GSPs used in the present study are based on our previous reports of tumor growth inhibitory effects of GSPs in non-melanoma and non-small cell lung cancer models $(3,9)$. The average body weights of mice which received control AIN76A or GSPs supplemented diet were comparable throughout the experimental protocol (Fig. 3A). The mice that were given GSPs in diet did not exhibit any physical sign of toxicity or abnormal behavior (data not shown). Weekly measurement of the tumor volume indicated that the average tumor growth in terms of tumor volume/mouse was lower in the GSPs-fed mice than the control diet group (Fig. 3B). As shown in Fig. 3B, on termination of the study at day 40 the average tumor volume in mice that received $0.2 \%$ GSPs and $0.5 \%$ GSPs in diet was, respectively, 35 and $52 \%$ less $(\mathrm{P}<0.01-0.001)$ than average volume of tumors from mice which were given control diet. The experiment was terminated at 40 days after tumor cell implantation. At this time the mice were sacrificed, the tumors harvested, and the wet weight of the tumor/mouse in each treatment group was recorded. As shown in Fig. 3C, the wet weight of the tumors was 36-60\% lower $(\mathrm{P}<0.01-0.001)$ in mice which were given GSPs $(0.2$ and 


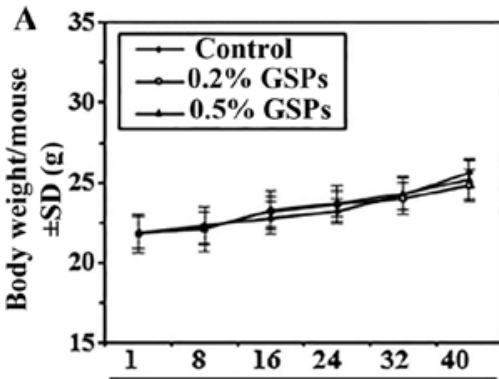

Days after tumor cell inoculation

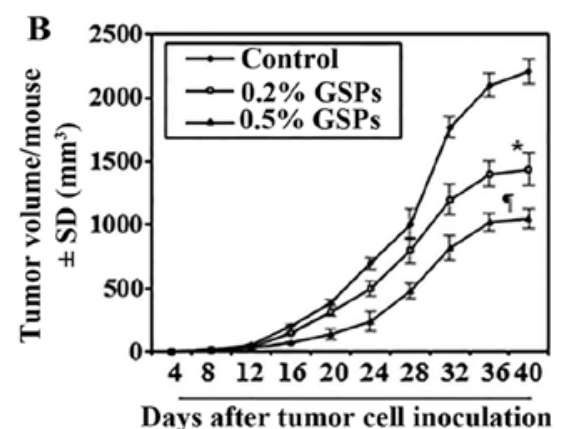

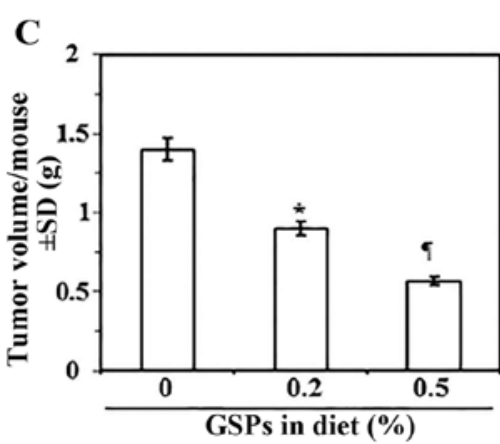

Figure 3. Dietary GSPs inhibit tumor xenograft growth of A375 melanoma cells grown in athymic nude mice. (A) Body weights of the mice in each treatment group were monitored on a weekly basis. The body weights of the GSP-treated ( 0.2 and $0.5 \%$ w/w) and non-GSP-treated mice did not significantly differ throughout the duration of the experiment, $n=5$. (B) Volume or size of tumor xenografts were recorded twice per week in each treatment group. Average tumor volume $\left(\mathrm{mm}^{3}\right) \pm \mathrm{SD}$ per mouse was calculated from each group and was plotted as a function of time. (C) Tumor xenograft tissues were harvested at the termination of the experiment at 40 days, and wet weight of the tumor per mouse in each group is reported as mean \pm SD (g). Significant inhibition vs. control group (non-GSP-treated), ${ }^{*} \mathrm{P}<0.01$ and ${ }^{\mathrm{I}} \mathrm{P}<0.001, \mathrm{n}=5 /$ group.

A
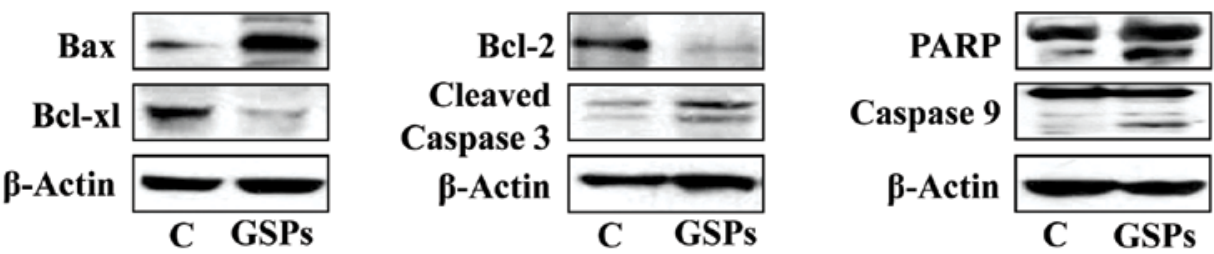

B
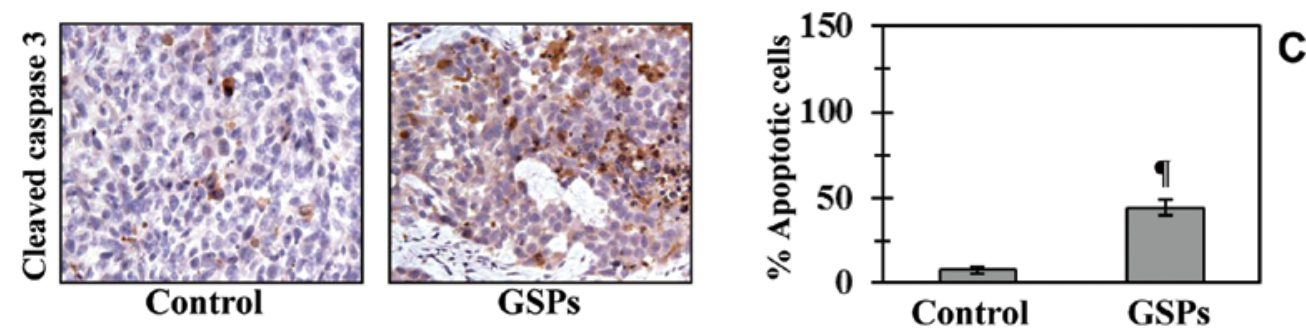

D

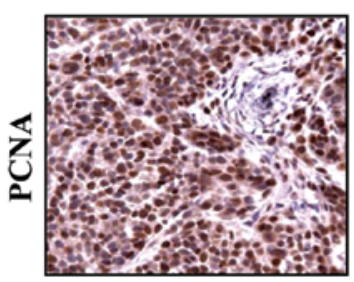

Control
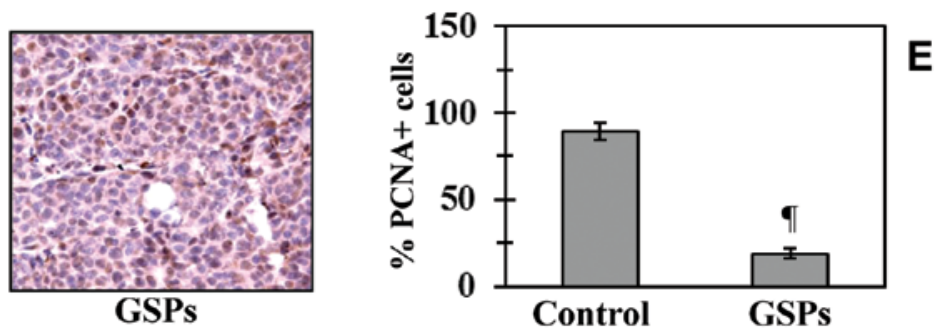

$\mathbf{F}$
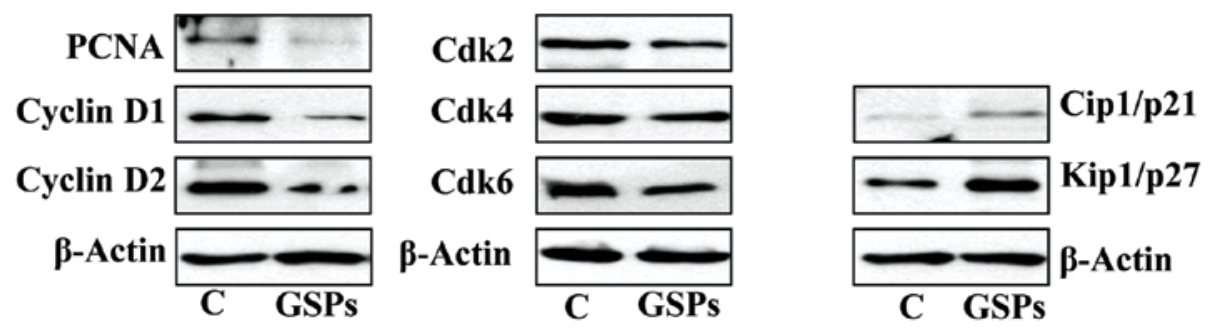

Figure 4. Dietary GSPs induce apoptosis and inhibit tumor cell proliferation in tumor xenograft tissues. Experiment was terminated at 40 days post inoculation of A375 cells, xenograft tissues were harvested for the analysis of biochemical markers. (A) Western blot analysis of proteins of apoptotic family in tumor lysates of control mice and mice that were fed on diet supplemented with $0.5 \%$ GSPs. (B) Representative photomicrographs showing immuno-histochemical detection of cleaved caspase- $3^{+}$cells in xenograft sections. (C) Number of cleaved caspase- $3^{+}$cells (apoptotic cells) were counted under a microscope at 3-4 different places of a section. (D) Representative photomicrographs are presented showing the PCNA ${ }^{+}$cells in different treatment groups. PCNA ${ }^{+}$cells are shown in dark brown. (C and E) Immunohistochemical staining data are presented as mean percentage of positive cells \pm SD, $n=3 / g$ roup. Significant difference vs. non-GSPs-treated control group; ${ }^{9} \mathrm{P}<0.001$. (F) Western blot analysis of the proteins of cell cycle regulation, PCNA and tumor suppressors in tumor lysates of control and GSPs-treated groups. 

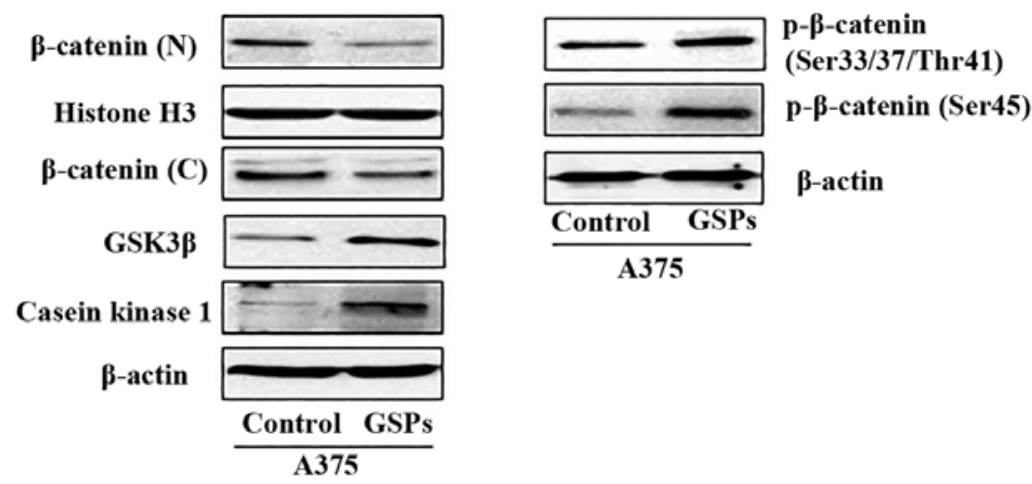

Figure 5. Dietary GSP-induced inhibition of melanoma tumor xenograft growth is associated with reduction in levels of cellular $\beta$-catenin. Western blot analysis shows the effect of dietary GSPs on cellular levels of $\beta$-catenin and expression of kinases regulating stabilization of $\beta$-catenin in A375 tumor xenograft tissues. N, nuclear; C, cytoplasmic.

$0.5 \%, \mathrm{w} / \mathrm{w})$ supplemented diet as compared to the tumors from mice given control AIN76A diet. As the chemotherapeutic effect of $0.5 \%$ GSP-supplemented diet on tumor growth was $>0.2 \%$ GSPs in diet, the tumor xenografts tissues from $0.5 \%$ GSP-treated group were subsequently used for comparison of biochemical parameters with the tumor xenografts from control AIN76A diet-fed group.

Dietary GSPs enhance apoptotic cell death of melanoma cells in tumor xenograft tissues. To determine whether GSP-induced inhibition of tumor xenograft growth is due to induction of apoptosis in cancer cells, we determined the effect of GSPs on the markers of apoptosis in xenograft samples. Western blot analysis revealed that tumor xenografts of mice which were given GSPs showed enhanced expression of proapoptotic protein Bax while the expression of anti-apoptotic proteins Bcl-2 and Bcl-xl were decreased compared with tumor xenografts of mice which were given control AIN76A diet (Fig. 4A). Compared to tumor xenografts of the control diet group, the enhanced expression of caspase-3, caspase- 9 and cleavage of PARP proteins was also observed in the melanoma tumor xenograft of mice given GSPs in diet (Fig. 4A). The proapoptotic effects of GSPs were further confirmed by immunohistochemical detection of activated caspase-3-positive cells in tumor xenograft samples (Fig. 4B). The percentage of activated caspase-3-positive cells in tumor samples from mice that were given GSPs was significantly higher $(\mathrm{P}<0.001)$ than the percentage of caspase-3-positive cells in the tumors of the untreated control mice (Fig. 4C).

Dietary GSPs inhibit the proliferative potential and the expression of cell cycle regulatory proteins of $G 0 / G 1$ phase in melanoma tumor xenograft tissues. Uncontrolled proliferation of tumor cells is a characteristic feature of most cancers. Therefore, to explain the growth inhibitory effect of dietary GSPs on tumor xenografts, we analyzed the melanoma tumor xenografts for the potential antiproliferative effects of GSPs using immunohistochemical detection of PCNA-positive cells. The results of the immunohistochemical detection of PCNA-positive cells in tumor xenograft tissues indicated that the percentage of proliferating cells was significantly reduced $(78 \%, \mathrm{P}<0.001)$ in tumor xenografts from mice given GSP-supplemented diet than tumor xenografts of
GSP-untreated control mice (Fig. 4D and E). Anti-proliferative effects of GSPs were further verified by western blot analysis for PCNA and markers of cell cycle regulatory proteins, such as cyclins (D1 and D2) and Cdks. Western blot analysis revealed that the levels of PCNA, cyclins and Cdks were lower in tumor xenografts of GSP-treated mice compared with tumor xenografts from control mice (Fig. 4F). Additionally, the expression levels of tumor suppressor proteins, Cip1/ p21 and Kip1/p27, were restored or reactivated in tumors of GSP-treated mice than the tumors from mice which were given control diet (Fig. 4F, right panel).

GSPs reduce $\beta$-catenin levels in melanoma tumor xenografts. As $\beta$-catenin has been involved in tumor progression, we determined whether dietary GSPs have any effect on the expression level of $\beta$-catenin in melanoma tumor xenografts. As shown in Fig. 5, western blot analysis revealed that treatment of GSPs reduced the nuclear as well as cytosolic levels of $\beta$-catenin in tumor xenograft samples. Similarly, the phosphorylation of $\beta$-catenin at $\mathrm{Ser}^{45}$, and other target residues $\left(\operatorname{Ser}^{33} / \mathrm{Ser}^{37} / \mathrm{Thr}^{41}\right)$, and the levels of CK $1 \alpha$ and GSK-3 $\beta$ were higher in tumor xenografts of mice which received GSPs in diet than the tumor xenografts of control mice.

$\beta$-catenin level in human melanoma cells determines the rate of melanoma cell growth. To further verify the role of $\beta$-catenin in melanoma cell growth, in vitro experiments were conducted. For this purpose, we knocked-down $\beta$-catenin in Mel1241 cells using $\beta$-catenin specific siRNA kit (Santa Cruz Biotechnology), and this led to reduced expression of $\beta$-catenin compared with scrambled RNA transfected control Mel1241 cells, as analyzed by western blot analysis (Fig. 6A). To determine whether the knockdown of $\beta$-catenin affects cell proliferation, control Mel1241 cells and $\beta$-catenin knockdown Mel1241 cells were cultured for 24 and $48 \mathrm{~h}$. Then, cells were harvested and counted under microscope using hemocytometer. As shown in Fig. 6B, knockdown of $\beta$-catenin was associated with reduced proliferation. The number of $\beta$-catenin knockdown Mel1241 cells was significantly $(44 \%, \mathrm{P}<0.01)$ less than the control Mel1241 cells after $48 \mathrm{~h}$ of culture. In another experiment, we examined the effect of $\beta$-catenin forced-overexpression in Mel1011 cells ( $\beta$-catenin inactivated cell line) by transfecting the cells with pcDNA3- $\beta$-catenin ${ }^{\mathrm{S} 33 \mathrm{Y}}$ plasmid. 
A

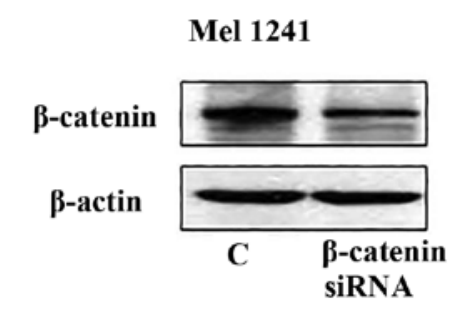

C

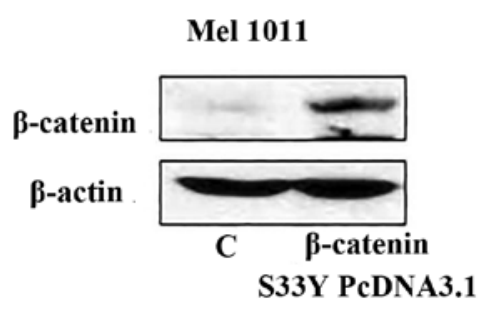

B

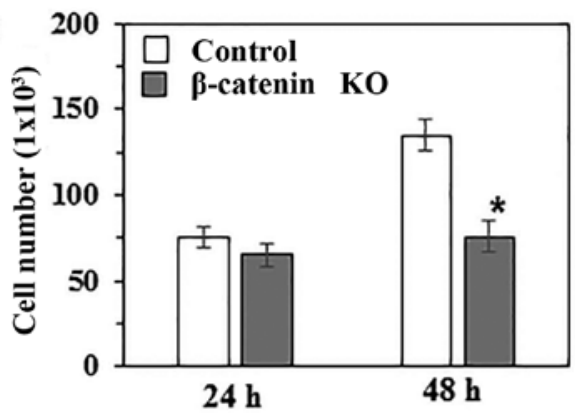

D

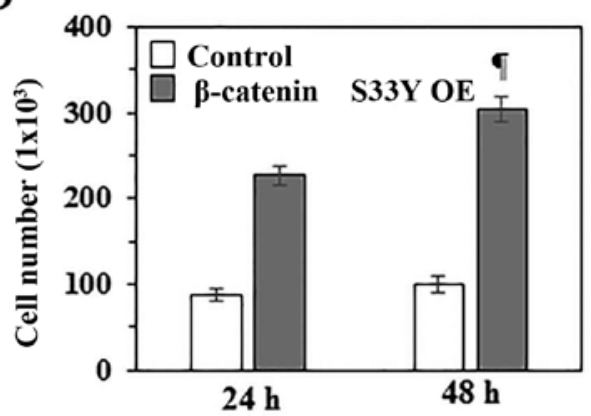

Figure 6. Effect of $\beta$-catenin manipulation on proliferation potential of melanoma cells. (A and B) The siRNA knockdown of $\beta$-catenin in Mel1241 cells (which have activated $\beta$-catenin) inhibits melanoma cell growth. $\beta$-catenin was knocked down in Mel1241 cells using siRNA transfection protocol. Forty-eight hours after the transfection of scrambled and $\beta$-catenin siRNA, Mel1241 cells (both control and $\beta$-catenin knock out) were harvested and plated in a 6 -well plate $(50,000 /$ well). Cells were harvested at 24 and $48 \mathrm{~h}$ time-points and were counted under a microscope using hemocytometer. (A) Detection of $\beta$-catenin level in scrambled and siRNA knockdown of Mel1241 cells using western blotting. (C) Overexpression of $\beta$-catenin ${ }^{\mathrm{S} 33 \mathrm{Y}}$ in Mel1011 cells enhances the rate of proliferation of Mel1011 cells. Mel1011 cells lack activated $\beta$-catenin. Verification by western blot analysis. (D) Forced expression of $\beta$-catenin in Mel1011 cells significantly enhances its proliferation potential compared to scrambled-treated cells, ${ }^{*} \mathrm{P}<0.01, \mathrm{n}=3$.

Forced overexpression of degradation resistant mutant form of $\beta$-catenin $\left(\beta\right.$-catenin $S^{33 \mathrm{Y}}$ ) was confirmed by western blotting using anti-FLAG antibody (Fig. 6C). Cells were cultured for 24 and $48 \mathrm{~h}$, thereafter cells were harvested and counted under a microscope. It was observed that forced overexpression of mutant $\beta$-catenin in Mel1011 cells resulted in increased cell proliferation rate compared with proliferation rate of control non-transfected Mel1011 cells. As shown in Fig. 6D, after $48 \mathrm{~h}$ of culture the number of $\beta$-catenin overexpressing Mel1011 cells was significantly higher $(67 \%, \mathrm{P}<0.001)$ than control Mel1011 cells.

Effect of dietary GSPs on tumor xenograft growth of $\beta$-catenin-proficient (active) and $\beta$-catenin-deficient (inactive) melanoma cells. As inhibition of melanoma xenograft growth by GSPs is associated with reduced levels of $\beta$-catenin, we further determined and compared the efficacy of dietary GSPs $(0.5 \%)$ on the tumor xenograft growth of melanoma cells with a difference in Wnt/ $\beta$-catenin activity (Me1928 vs. Mel1011) and those cells were subcutaneously implanted $\left(2 \times 10^{6}\right.$ cells/ mouse) in nude mice. Experiment was continued for 49 days based on IACUC guidelines. As shown in Fig. 7A (left panel), significant inhibition $(\mathrm{P}<0.001)$ in tumor xenograft growth was observed in mice which were implanted with Mel928 cells ( $\beta$-catenin proficient) and fed with GSPs-supplemented diet. At the termination of the experiment at day 49 the average tumor volume in the mice which received $0.5 \%$ GSP supplemented diet was $47 \%$ less $(\mathrm{P}<0.001)$ compared with the tumor volume of Mel928 tumor xenografts of mice which received control AIN76A diet (Fig. 7A). However, GSPs failed to inhibit the xenograft growth of Mel1011 cells ( $\beta$-catenin-deficient) in an identical experimental protocol (Fig. 7A). Additionally, the tumor xenograft volume of Mel928 cells was greater than tumor xenograft volume of Mel1011 at the termination of the experiment. The wet weights of the tumors from each treatment group at the termination of the experiment were recorded (Fig. 7B). It was observed that: i) dietary GSPs resulted in significant reduction in weight $(39 \%, \mathrm{P}<0.01)$ of $\mathrm{Mel} 928$ tumor xenografts. In contrast, GSPs did not have significant inhibitory effect on the weight of tumor xenografts of Mel1011 cells, which have inactivated $\beta$-catenin. ii) The wet weight of Me1928 tumor xenografts recorded after 49 days of protocol was higher than the wet weight of Mel1011 tumor xenografts. Additionally, western blot analysis revealed that dietary GSPs reduced the expression of PCNA and increased expression of activated caspase-3 in Mel928 tumor xenografts, whereas this effect of GSPs was not observed in tumor xenografts of Mel1011 cells (Fig. 7C).

\section{Discussion}

The antitumor activity of GSPs has been shown in some preclinical models (3-11), however, the antitumor activity of GSPs has not been explored in melanoma. We therefore determined the therapeutic effects of GSPs on melanoma cell lines using both in vitro and in vivo models. Here, we report that GSPs significantly decrease the viability and induce apoptotic cell death of human melanoma cell lines, which are $B R A F$-mutated (A375) and non-BRAF-mutated but highly specific to metastasis (Hs294t). Notably, GSPs did not 


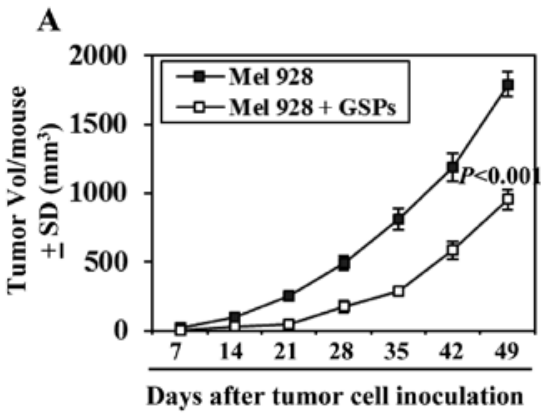

B

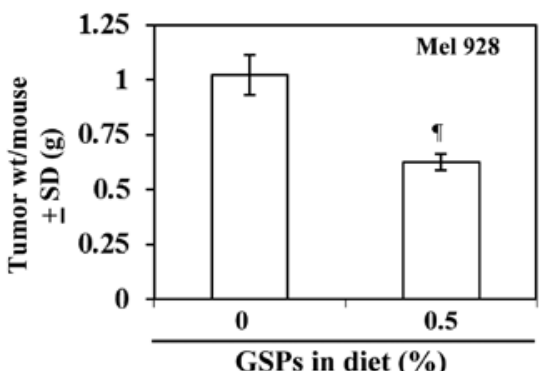

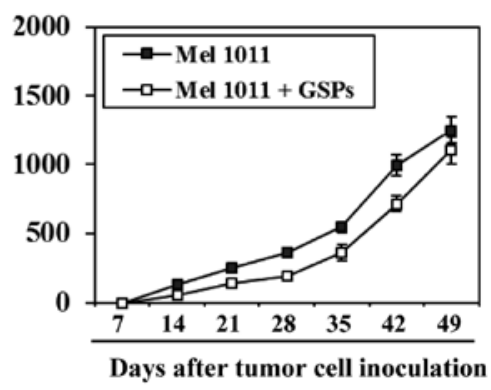

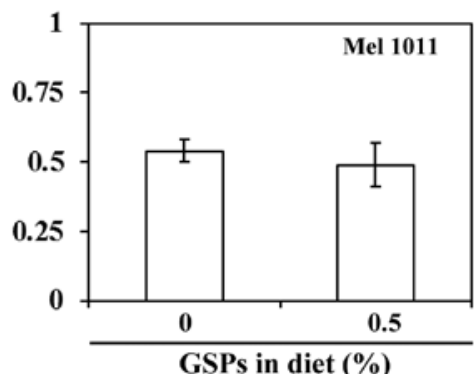

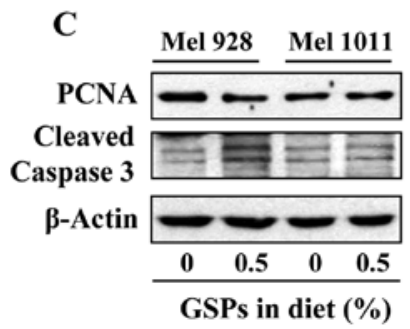

Figure 7. Effect of dietary GSPs on the growth of $\beta$-catenin activated and $\beta$-catenin inactivated melanoma tumor xenograft in athymic nude mice. Mel928 (activated $\beta$-catenin) and Mel1011 ( $\beta$-catenin inactivated) cells were subcutaneously implanted in athymic nude mice and the effect of dietary GSPs was determined on weekly basis. (A) The volumes of tumor xenograft was recorded in each treatment group once per week. Average tumor volume $\left(\mathrm{mm}^{3}\right) \pm \mathrm{SD}$ was plotted as a function of time. (B) Tumor xenograft tissues were harvested at the termination of the experiment at day 49, and wet weight of the tumor per mouse in each group is reported as mean $\pm \mathrm{SD}(\mathrm{g}), \mathrm{n}=5$. Significant difference vs. non-GSP-treated control group, ${ }^{\mathrm{g}} \mathrm{P}<0.01$. (C) Effect of dietary GSPs on the levels of PCNA and cleaved caspase-3 in the treatment groups of both Me1928 and Mel1011 tumor xenografts. Tumor lysates were used for western blot analysis.

exhibit significant cytotoxicity to normal human epidermal melanocytes under identical experimental conditions. A major apoptotic signal transduction cascade associated with induction of apoptosis includes the proteins of Bcl-2 family, which either promote cell survival or promote apoptosis $(25,26)$. We found that treatment of A375 and Hs294t cells with GSPs resulted in a dose-dependent decrease in the levels of anti-apoptotic proteins (Bcl-2, Bcl-xl) and a simultaneous increase in the pro-apoptotic protein (Bax). It is well known that the cleavage of caspases and PARP contributes in cancer cell apoptosis. Our data suggest that GSPs-induced apoptosis in melanoma cells is mediated through activation of caspase-3, caspase-9 and PARP, and this may be a possible mechanism of GSP-induced apoptosis in melanoma cells.

The molecular mechanisms underlying the progression of melanoma remain unresolved. Various studies have implicated constitutively active $\mathrm{Wnt} / \beta$-catenin signaling in melanoma progression and metastasis $(12,13)$. Nuclear $\beta$-catenin accumulation has been correlated with late stages of tumor progression. The presence of mutated $\beta$-catenin is associated with aggressive tumor growth and regulates expression of various target genes that mediate cellular processes including proliferation and migration $(27,28)$. In the canonical model of Wnt signaling, $\beta$-catenin is phosphorylated at certain key residues by GSK-3 $\beta$ and casein kinase $1 \alpha(\mathrm{CK} 1 \alpha)$ leading to its ubiquitination and subsequent degradation (24). Like cancers of other organs, the regulation of $\beta$-catenin is lost in melanoma (29-31). This then leads to nuclear accumulation of $\beta$-catenin and subsequent stimulation of downstream target genes, which includes the genes of cell proliferation (e.g., PCNA, cyclins and cyclin-dependent kinases) and tumor progression (e.g., matrix metalloproteinases) (32-34). Our results show that inhibition of melanoma cell growth by GSPs is associated with the reduction in the accumulation of nuclear and cytosolic $\beta$-catenin in melanoma cells. It has been shown that phosphorylation of $\beta$-catenin at critical target residues (such as at $\mathrm{Ser}^{45}, \mathrm{Ser}^{33 / 37}$ and $\mathrm{Thr}^{41}$ ) by GSK-3 $\beta$ and CK1 $\alpha$ within the cytosolic destruction complex leads to degradation of $\beta$-catenin and thus reduces its nuclear accumulation (24). In the present study, we found that treatment of melanoma cells with GSPs enhances the expression of GSK-3 $\beta$ and CK1 $\alpha$, and $\beta$-catenin is phosphorylated at critical target residues. This effect then leads to degradation of $\beta$-catenin within the degradation complex resulting in its reduced nuclear and cytosolic accumulation. It thus explains inhibitory effects of GSPs against melanoma cell growth. 
To verify therapeutic effects of GSPs obtained in in vitro cell culture system, in vivo studies were conducted using in vivo tumor xenograft model. The GSPs were administered in the diet of the mice as this approach was proven effective in other cancers $(4,8-11)$. The present study provides evidence that dietary administration of GSPs inhibits the growth of A375 tumor xenografts in the athymic nude mice without any apparent sign of toxicity. The identification of molecular targets is an important consideration in terms of monitoring the clinical efficacy of GSPs in suggesting potential combinations with other agents or drugs. In this context, the inhibitory effect of dietary GSPs on the growth of tumor xenograft in athymic nude mice was studied and found to be associated with the: i) induction of apoptotic cell death of tumor cells, as indicated by the analysis of the proteins of Bcl-2 family and activated caspase- 3 and PARP proteins; ii) inhibition of PCNA; iii) control of cell cycle regulatory proteins; and iv) reduction in the levels of cytosolic and nuclear $\beta$-catenin in tumor xenograft samples at the termination of the in vivo animal experiments. In an attempt to further verify the role of GSPs in melanoma growth by targeting $\beta$-catenin, the siRNA knockdown of $\beta$-catenin in Mel1241 cells ( $\beta$-catenin-activated cell line) resulted in suppression of cell proliferation, while forced expression of $\beta$-catenin in Mel1011 cells ( $\beta$-catenininactivated) resulted in enhanced proliferation rate of these melanoma cells. These observations were further supported by another in vivo tumor xenograft experiment in which Mel928 ( $\beta$-catenin activated) and Mel1011 ( $\beta$-catenin-inactivated) cells were subcutaneously implanted and mice given AIN76A control diet with and without supplementation of GSPs $(0.5 \%$, w/w). Administration of dietary GSPs significantly inhibited the growth of Mel928 melanoma tumor xenografts, but failed to inhibit the xenograft growth of Mel1011, thus, further supporting our hypothesis that inhibition of melanoma growth by dietary GSPs is mediated through inhibition of accumulation of $\beta$-catenin in melanoma cells.

In summary, the outcome of this study suggests that GSPs have the ability to block or inhibit the growth potential of melanoma cells, and this effect of GSPs is mediated through targeting $\beta$-catenin and its signaling molecules in melanoma. Thus intervention strategies targeting key molecules of the Wnt/ $\beta$-catenin pathway may represent promising strategies to inhibit the growth and progression of melanoma. This new insight into the anti-melanoma activity of GSPs could serve as the basis for alternative therapy of malignant melanoma as a single agent or as a combination with already known drugs of melanoma in high risk individuals.

\section{Acknowledgements}

The present study was supported by grants from the National Institutes of Health (NIH, CA166883) and the Veterans Administration Merit Review Award (1I01BX001410) to S.K.K.

\section{References}

1. Hall HI, Miller DR, Rogers JD and Bewerse B: Update on the incidence and mortality from melanoma in the United States. J Am Acad Dermatol 40: 35-42, 1999.
2. Strouse JJ, Fears TR, Tucker MA and Wayne AS: Pediatric melanoma: Risk factor and survival analysis of the surveillance, epidemiology and end results database. J Clin Oncol 23: 4735-4741, 2005

3. Mittal A, Elmets CA and Katiyar SK: Dietary feeding of proanthocyanidins from grape seeds prevents photocarcinogenesis in SKH-1 hairless mice: Relationship to decreased fat and lipid peroxidation. Carcinogenesis 24: 1379-1388, 2003.

4. Sharma SD, Meeran SM and Katiyar SK: Dietary grape seed proanthocyanidins inhibit UVB-induced oxidative stress and activation of mitogen-activated protein kinases and nuclear factor-kappaB signaling in in vivo SKH-1 hairless mice. Mol Cancer Ther 6: 995-1005, 2007.

5. Nandakumar V, Singh T and Katiyar SK: Multi-targeted prevention and therapy of cancer by proanthocyanidins. Cancer Lett 269: 378-387, 2008.

6. Vaid M, Singh T and Katiyar SK: Grape seed proanthocyanidins inhibit melanoma cell invasiveness by reduction of PGE2 synthesis and reversal of epithelial-to-mesenchymal transition. PLoS One 6: e21539, 2011.

7. Sun Q, Prasad R, Rosenthal E and Katiyar SK: Grape seed proanthocyanidins inhibit the invasive potential of head and neck cutaneous squamous cell carcinoma cells by targeting EGFR expression and epithelial-to-mesenchymal transition. BMC Complement Altern Med 11: 134-145, 2011.

8. Meeran SM, Vaid M, Punathil T and Katiyar SK: Dietary grape seed proanthocyanidins inhibit $12-O$-tetradecanoyl phorbol13 -acetate-caused skin tumor promotion in 7,12-dimethylbenz $[a]$ anthracene-initiated mouse skin, which is associated with the inhibition of inflammatory responses. Carcinogenesis 30: $520-528,2009$.

9. Akhtar S, Meeran SM, Katiyar N and Katiyar SK: Grape seed proanthocyanidins inhibit the growth of human non-small cell lung cancer xenografts by targeting insulin-like growth factor binding protein-3, tumor cell proliferation, and angiogenic factors. Clin Cancer Res 15: 821-831, 2009.

10. Prasad R, Vaid M and Katiyar SK: Grape proanthocyanidin inhibit pancreatic cancer cell growth in vitro and in vivo through induction of apoptosis and by targeting the PI3K/Akt pathway. PLoS One 7: e43064, 2012.

11. Prasad R and Katiyar SK: Bioactive phytochemical proanthocyanidins inhibit growth of head and neck squamous cell carcinoma cells by targeting multiple signaling molecules. PLoS One 7: e46404, 2012.

12. Sinnberg T, Menzel M, Kaesler S, Biedermann T, Sauer B, Nahnsen S, Schwarz M, Garbe C and Schittek B: Suppression of casein kinase 1alpha in melanoma cells induces a switch in beta-catenin signaling to promote metastasis. Cancer Res 70: 6999-7009, 2010.

13. Syed DN, Afaq F, Maddodi N, Johnson JJ, Sarfaraz S, Ahmad A Setaluri V and Mukhtar H: Inhibition of human melanoma cell growth by the dietary flavonoid fisetin is associated with disruption of Wnt/ $\beta$-catenin signaling and decreased Mitf levels. J Invest Dermatol 131: 1291-1299, 2011.

14. Lucero OM, Dawson DW, Moon RT and Chien AJ: A re-evaluation of the 'oncogenic' nature of Wnt/beta-catenin signaling in melanoma and other cancers. Curr Oncol Rep 12: 314-318, 2010.

15. Delmas V, Beermann F, Martinozzi S, Carreira S, Ackermann J, Kumasaka M, Denat L, Goodall J, Luciani F, Viros A, et al: Betacatenin induces immortalization of melanocytes by suppressing p16INK4a expression and cooperates with N-Ras in melanoma development. Genes Dev 21: 2923-2935, 2007.

16. Chien AJ, Moore EC, Lonsdorf AS, Kulikauskas RM, Rothberg BG, Berger AJ, Major MB, Hwang ST, Rimm DL and Moon RT: Activated Wnt/beta-catenin signaling in melanoma is associated with decreased proliferation in patient tumors and a murine melanoma model. Proc Natl Acad Sci USA 106: 1193-1198, 2009.

17. Maelandsmo GM, Holm R, Nesland JM, Fodstad $\varnothing$ and Flørenes VA: Reduced beta-catenin expression in the cytoplasm of advanced-stage superficial spreading malignant melanoma. Clin Cancer Res 9: 3383-3388, 2003.

18. Kageshita T, Hamby CV, Ishihara T, Matsumoto K, Saida T and Ono T: Loss of beta-catenin expression associated with disease progression in malignant melanoma. Br J Dermatol 145: 210-216, 2001.

19. Bachmann IM, Straume O, Puntervoll HE, Kalvenes MB and Akslen LA: Importance of P-cadherin, beta-catenin, and Wnt5a/ frizzled for progression of melanocytic tumors and prognosis in cutaneous melanoma. Clin Cancer Res 11: 8606-8614, 2005. 
20. Mantena SK, Sharma SD and Katiyar SK: Berberine inhibits growth, induces G1 arrest and apoptosis in human epidermoid carcinoma A431 cells by regulating Cdki-Cdk-cyclin cascade, disruption of mitochondrial membrane potential and cleavage of caspase 3 and PARP. Carcinogenesis 27: 2018-2027, 2006.

21. Vaid M, Sharma SD and Katiyar SK: Honokiol, a phytochemical from the Magnolia plant, inhibits photocarcinogenesis by targeting UVB-induced inflammatory mediators and cell cycle regulators: Development of topical formulation. Carcinogenesis 31: 2004-2011, 2010.

22. Adams JM and Cory S: Life-or-death decisions by the Bcl-2 protein family. Trends Biochem Sci 26: 61-66, 2001.

23. Chao DT and Korsmeyer SJ: BCL-2 family: Regulators of cell death. Annu Rev Immunol 16: 395-419, 1998.

24. Liu C, Li Y, Semenov M, Han C, Baeg GH, Tan Y, Zhang Z, Lin $\mathrm{X}$ and $\mathrm{He} \mathrm{X}$ : Control of beta-catenin phosphorylation/ degradation by a dual-kinase mechanism. Cell 108: 837-847, 2002.

25. Gross A, McDonnell JM and Korsmeyer SJ: BCL-2 family members and the mitochondria in apoptosis. Genes Dev 13: 1899-1911, 1999.

26. Hockenbery D, Nuñez G, Milliman C, Schreiber RD and Korsmeyer SJ: Bcl-2 is an inner mitochondrial membrane protein that blocks programmed cell death. Nature 348: 334-336, 1990.

27. Klaus A and Birchmeier W: Wnt signalling and its impact on development and cancer. Nat Rev Cancer 8: 387-398, 2008.

28. Gavert $\mathrm{N}$ and Ben-Ze'ev A: $\beta$-Catenin signaling in biological control and cancer. J Cell Biochem 102: 820-828, 2007.
29. Rimm DL, Caca K, Hu G, Harrison FB and Fearon ER: Frequent nuclear/cytoplasmic localization of beta-catenin without exon 3 mutations in malignant melanoma. Am J Pathol 154: 325-329, 1999.

30. Rubinfeld B, Robbins P, El-Gamil M, Albert I, Porfiri E and Polakis P: Stabilization of beta-catenin by genetic defects in melanoma cell lines. Science 275: 1790-1792, 1997.

31. Demunter A, Libbrecht L, Degreef H, De Wolf-Peeters C and van den Oord JJ: Loss of membranous expression of beta-catenin is associated with tumor progression in cutaneous melanoma and rarely caused by exon 3 mutations. Mod Pathol 15: 454-461, 2002.

32. Lowy AM, Clements WM, Bishop J, Kong L, Bonney T, Sisco K, Aronow B, Fenoglio-Preiser C and Groden J: $\beta$-Catenin/Wnt signaling regulates expression of the membrane type 3 matrix metalloproteinase in gastric cancer. Cancer Res 66: 4734-4741, 2006.

33. Li YJ, Wei ZM, Meng YX and Ji XR: Beta-catenin up-regulates the expression of cyclinD1, c-myc and MMP-7 in human pancreatic cancer: Relationships with carcinogenesis and metastasis. World J Gastroenterol 11: 2117-2123, 2005

34. Qi J, Chen N, Wang J and Siu CH: Transendothelial migration of melanoma cells involves $\mathrm{N}$-cadherin-mediated adhesion and activation of the beta-catenin signaling pathway. Mol Biol Cell 16: 4386-4397, 2005. 